\title{
Effect of the third monomer unit on the phase transition of oriented ethylene-tetrafluoroethylene copolymer studied by the temperature-dependent measurements of 2D X-ray scattering and polarized infrared spectroscopy
}

\begin{abstract}
Atsushi Funaki ${ }^{1}$, Suttinun Phongtamrug ${ }^{2}$ and Kohji Tashiro ${ }^{3}$
The influence of third monomer units with different chain lengths on the phase-transition behavior of the uniaxially oriented ethylene tetrafluoroethylene (ETFE)-alternating copolymer has been investigated through the use of temperature-dependent measurements of X-ray fiber diagrams, small-angle X-ray scattering patterns and polarized infrared spectra. (i) The phase transition between the low- and high-temperature phases occurs discontinuously; (ii) in the transition, the conformational disordering and radical thermal motion of the chains occur drastically as known from the increment of the half-width of the $\mathrm{X}$-ray 004 reflection profile in the high-temperature region, which is associated with the trans-gauche conformational disordering; (iii) the phase-transition temperature decreases with an increase in the third monomer unit content, and the decreasing rate is overwhelmingly higher for the terpolymer with short $\mathrm{CF}_{3}$ groups than for the terpolymer with longer $\mathrm{C}_{4} \mathrm{~F}_{9}$ groups. The $\mathrm{CF}_{3}$ groups are included in the crystal lattice and affect the phase-transition point significantly, whereas the $\mathrm{C}_{4} \mathrm{~F}_{9}$ groups are expelled from the lattice and affect the transition behavior to a small degree; and (iv) The temperature-dependent two-dimensional small-angle X-ray scattering pattern measurements of the oriented samples revealed that the long period of the stacked lamellar structure did not change in the transition between the low- and high-temperature phases.
\end{abstract}

Polymer Journal (2013) 45, 545-554; doi:10.1038/pj.2012.177; published online 14 November 2012

Keywords: ethylene-tetrafluoroethylene copolymer; phase transition; polarized infrared spectra; small-angle X-ray scattering; stacked lamellar structure; third monomer unit; wide-angle X-ray diffraction

\section{INTRODUCTION}

The ethylene tetrafluoroethylene (ETFE)-alternating copolymer is a melt-processable fluororesin. Because of its excellent thermal and chemical durability, as well as its electrical properties, ETFE is used for various industrial applications, including chemical manufacturing materials, wire coating insulation and so on. ${ }^{1}$ This polymer film is also used as a covering sheet for sports facilities because of its excellent weatherability, transparency and mechanical strength. ${ }^{2}$ These various properties are sensitively governed by the structure and properties of the crystal lattice and the higher-order structure, which is composed of both crystalline and amorphous regions.

The ETFE copolymer is known to experience a crystalline phase transition between its low-temperature and high-temperature phases. In the low-temperature phase, the essentially planar zigzag chains pack within the triclinic unit cell, as reported in our earlier study. ${ }^{3}$ In the temperature region approximately $60^{\circ} \mathrm{C}$, ETFE transforms to its pseudo-hexagonal, high-temperature phase. The phase-transition behavior is sensitively affected by the ethylene (E)/tetrafluoroethylene (TFE) molar ratio. ${ }^{4-6}$ When the TFE content is increased, the crystal structure transforms from the triclinic to the pseudo-hexagonal form, even at an ambient temperature. ${ }^{4-6}$

The phase-transition behavior was also reported to be remarkably affected by the introduction of a third monomer unit (the termonomer). Originally, the third component was introduced to solve the problems encountered in actual applications. When the ETFE copolymer molds are constrained by the application of high stress at high temperatures, cracks develop easily during the molding process. By introducing the third monomer unit into the ETFE copolymer chains, the appearance of the thermal-stress cracking phenomenon is drastically reduced. ${ }^{7}$ Therefore, most of the

${ }^{1}$ AGC Chemicals, Asahi Glass Co., Ltd, Yokohama, Japan; ²Faculty of Applied Science, King Mongkut's University of Technology North Bangkok, Bangkok, Thailand and ${ }^{3}$ Department of Future Industry-oriented Basic Science and Materials, Toyota Technological Institute, Nagoya, Japan 
commercially available ETFE copolymers are three-component copolymers that include small amounts of the third (or sometimes the fourth) monomer units. There have been a limited number of structural studies performed on three-component ETFE copolymers (or terpolymers). For example, the study of a terpolymer consisting of $\mathrm{E}$, TFE and perfluoropropylvinylether $\left(\mathrm{CF}_{2}=\mathrm{CFOCF}_{2} \mathrm{CF}_{2} \mathrm{CF}_{3}\right)$ demonstrated that the high-temperature, pseudo-hexagonal phase can also appear at room temperature; in addition, the crystallinity is reduced, and the crystallite size is smaller than that of the ETFE twocomponent system. ${ }^{5}$ However, the details have not yet been sufficiently clarified.

In our previous publication, the role of the third monomer unit on the phase-transition behavior of the ETFE copolymer was investigated using unoriented samples. ${ }^{8}$ In particular, to clarify the effects of side-branching length and content of the third monomer unit on the phase-transition behavior of the ETFE copolymer, nonafluoro-1-hexene $\left(\mathrm{CH}_{2}=\mathrm{CH}\left(\mathrm{CF}_{2}\right)_{3} \mathrm{CF}_{3}\right)$ and hexafluoropropylene $\left(\mathrm{CF}_{2}=\mathrm{CFCF}_{3}\right)$ were chosen as the third monomer unit, for which these units possessed different side-branch lengths, $-\left(\mathrm{CF}_{2}\right)_{3} \mathrm{CF}_{3}$ and $-\mathrm{CF}_{3}$, respectively. For the ETFE copolymer containing nonafluoro1-hexene (here named ET-C4F) copolymer, the crystal phasetransition temperature was almost the same in the termonomer range of $0.7-3.3 \mathrm{~mol} \%$. For the ETFE copolymer containing hexafluoropropylene of ET-CF3 copolymer, however, the crystal phase-transition temperature decreased drastically with a slight increase in the hexafluoropropylene content. These phenomena were related to the structural characteristics of the terpolymers: the short side chains $\left(-\mathrm{CF}_{3}\right)$ are included in the crystal lattice, whereas the long side chains $\left(-\mathrm{C}_{4} \mathrm{~F}_{9}\right)$ are excluded from the crystal lattices. However, these observations must be considered in a more quantitative manner. Unfortunately, the use of the unoriented samples limited the detailed analysis achievable with X-ray diffraction techniques because the X-ray reflections overlapped with each other, making the X-ray data analysis an ambiguous process. In the present study, two-dimensional (2D) wide-angle X-ray diffraction and polarized infrared spectra were measured for uniaxially oriented samples to separate the overlapping reflection profiles and to clarify the role of side chains in the phase transition more explicitly and extensively. The measurements of the $(00 l)$ reflections are useful for the investigation of conformational disordering in the molecular chains, and these measurements are performed for the first time by the use of oriented samples. In addition, the oriented samples are useful for the analysis of infrared spectral data. The highly overlapped infrared band profile may be clearly separated into its various components with the transition dipoles, both perpendicular and parallel to the chain axis, by the use of a polarizer, thereby simplifying the process necessary to trace the temperature dependence of the infrared band components, which are intrinsic to the crystalline trans and gauche forms. In addition to the temperature-dependent measurements of the wide-angle X-ray diffraction and fourier transform infrared spectra, 2D small-angle X-ray scattering measurements were also performed here for the first time, for the three different types of the copolymers identified in this study. The higher-order structure (or stacked lamellar structure) has been analyzed for these samples, from which the influence of the third monomer unit on the higher-order structure has been well clarified.

As mentioned above, it should be reemphasized that many previously established problems have been solved here for the first time; in particular, the effect of the third monomer unit was investigated in this study of the phase-transition behaviors of the three different types of ETFE copolymers by using oriented samples.

\section{EXPERIMENTAL PROCEDURE}

\section{Samples}

In this study, two different chemical species were introduced as the third monomer unit, including nonafluoro-1-hexene $\left(\mathrm{CH}_{2}=\mathrm{CH}\left(\mathrm{CF}_{2}\right)_{3} \mathrm{CF}_{3}\right)$ and hexafluoropropylene $\left(\mathrm{CF}_{2}=\mathrm{CFCF}_{3}\right)$. Copolymers consisting of $\mathrm{E}$, TFE and a third monomer unit were synthesized by means of a solution-polymerization method using fluorinated hydrocarbon as the solvent. ${ }^{9}$ The chemical compositions were analyzed by performing molten-state fluorine-19 nuclear magnetic resonance measurements and fluorine elemental analysis. The compositions of the synthesized copolymers are listed in Table 1.

The samples were melted and then cooled rapidly to room temperature. Next, the samples were stretched to approximately three times their original length at approximately $100^{\circ} \mathrm{C}$, followed by an annealing treatment at approximately $20^{\circ} \mathrm{C}$ below the melting temperature. The annealing treatment was carried out for $3 \mathrm{~h}$ under tension. The as-prepared, uniaxially oriented samples were used for the X-ray diffraction measurements. The oriented thin films measured approximately $8 \mu \mathrm{m}$ in thickness and were used for the infrared spectral measurements.

\section{Measurements}

The X-ray fiber diagrams were measured using a MAC Science DIP1000 X-ray diffraction (Yokohama, Japan) system with a graphite-monochromatized Mo$\mathrm{K} \alpha$ line $(\lambda=0.71073 \AA)$ as the incident $\mathrm{X}$-ray beam. An imaging plate was used as the $2 \mathrm{D}$ detector. The temperature-dependent measurements of the X-ray fiber diagrams were performed by blowing nitrogen gas onto the sample set, as mounted on the goniometer head, using a cryostat CryoMini Coldhead (Rigaku, Tokyo, Japan) and a Rigaku (Tokyo, Japan) temperature controller in the range of -120 to $160^{\circ} \mathrm{C}$. The temperature was monitored by a thermocouple, which was attached directly to the sample. The temperature fluctuations during analysis were within $\pm 2.0^{\circ} \mathrm{C}$ across the entire monitored region. The (004) reflection was measured in transmission mode using a Rigaku RINT-TTR3 plus X-ray diffractometer with a graphite-monochromatized $\mathrm{Cu}-\mathrm{K} \alpha$ line $(\lambda=1.5418 \AA)$ as the incident $\mathrm{X}$-ray beam. The temperature control was performed using the cryostat and each sample was set with the homemade equipment.

The SAXS patterns were measured using a Nanoviewer (Rigaku Co., Ltd.). The X-ray beam generated from a rotating copper anode $(\mathrm{Cu}-\mathrm{K} \alpha$ line $)$ was reflected on the confocal mirror to produce a highly brilliant X-ray beam of approximately $100 \mu \mathrm{m}$ in size on the sample position. The scattered X-ray signals were collected using a 2D detector Pilatus (Dectris, Baden, Switzerland) set at a distance of $760 \mathrm{~mm}$ from the sample position. A homemade heater was used for heating the sample, the temperature of which was monitored by a thermocouple contacted directly on the sample.

The polarized infrared spectra were measured for the uniaxially oriented samples at various temperatures using a homemade heater with a Varian FTS7000 Fourier-transform infrared spectrometer (Varian, Boston, MA, USA) equipped with a wire-grid polarizer. The resolution power was $2 \mathrm{~cm}^{-1}$.

\section{Table 1 Compositions of two- and three-component ETFE} copolymers

\begin{tabular}{lcccc}
\hline & \multicolumn{4}{c}{ Composition/mol\% } \\
\cline { 2 - 5 } Sample & $E$ & TFE & NFH & HFP \\
\hline ET & 46.0 & 54.0 & - & - \\
ET-C4F9(0.8\%) & 45.7 & 53.6 & 0.8 & - \\
ET-C4F9(1.5\%) & 45.4 & 53.2 & 1.5 & - \\
ET-C4F9(3.3\%) & 38.6 & 58.1 & 3.3 & - \\
ET-CF3(2.7\%) & 43.8 & 53.5 & - & 2.7 \\
ET-CF3(4.6\%) & 42.0 & 53.4 & - & 4.6 \\
\hline
\end{tabular}

Abbreviations: $\mathrm{E}$, ethylene; ETFE, ethylene tetrafluoroethylene; HFP, hexafluoropropylene $\mathrm{CF}_{2}=\mathrm{CFCF}_{3}$; NFH, 3,3,4,4,5,5,6,6,6-nonafluoro-1-hexene $\mathrm{CH}_{2}=\mathrm{CH}\left(\mathrm{CF}_{2}\right)_{3} \mathrm{CF}_{3}$; TFE, tetrafluoroethylene. 


\section{RESULTS AND DISCUSSION}

The $2 \mathrm{D}$ wide-angle $\mathrm{X}$-ray diffraction patterns

Figure 1 shows the temperature dependence of the X-ray fiber diagrams taken for the uniaxially oriented ET-C4F9(0.8\%) sample, as an example. The fiber period estimated from the interlayer spacings was $5.10 \AA$, indicating essentially a trans-zigzag chain conformation. ${ }^{3,10-12}$ The diffuse scattering was observed along the layer lines. In our previous study, ${ }^{3}$ the structure analysis was performed successfully for the ET two-component system: two planar zigzag chains are packed in parallel in the unit cell. The diffuse X-ray scattering pattern was determined to come from the statistically random shift of the neighboring chains along the chain axis. More specifically, the diffuse streaks along the horizontal lines come from the random shift in small domains consisting of the regular unit cells along the $c$-axis. The concept of structural disorder was proposed for the quantitative interpretation of the observed $(00 l)$ reflection profile. These structural characteristics were also observed for the other ETC4F9 copolymers, with nonafluoro-1-hexene contents of 1.5 and $3.3 \%$. Figure 2 shows the equatorial line profiles obtained from Figure 1 . In the low-temperature region, the inner-most reflections were detected at $2 \theta=8.2$ and $9.2^{\circ}(\mathrm{Mo}-\mathrm{K} \alpha)$, which are indexed as the 110 and 200 reflections of the triclinic cell, respectively. ${ }^{3}$ The lattice constants projected along the chain axis are evaluated as $\left(a^{\prime}\right.$, $\left.b^{\prime}\right)=(8.78,5.92 \AA),(8.84,5.82 \AA)$ and $(8.93,5.92 \AA)$, respectively for ET-C4F9 $(0.8 \%),(1.5 \%)$ and $(3.3 \%)$ at $-100{ }^{\circ} \mathrm{C}$ where the angle $\gamma=90^{\circ}$. With increasing temperature, these two reflections approached each other in an apparently continuous fashion and merged into one at approximately $50-100{ }^{\circ} \mathrm{C}$, as already reported for the unoriented samples. However, when the higher-angle region approximately $2 \theta=22^{\circ}$ is focused, the (130) reflection was found to coexist with a newly appearing reflection at approximately $2 \theta=23^{\circ}$ in the temperature range of $50-100^{\circ} \mathrm{C}$, which is assigned to the 120 plane of the high-temperature phase. In Figure 3, the lattice spacings of several reflections are plotted against the temperature, where the diffraction profiles given in Figure 2 were separated into the components (110 and 200) using a commercial software Grams/AI 8 (Thermofischer Scientific Com., Waltham, MA, USA), and the peak position and integrated intensity were evaluated individually. As the temperature was further increased, the reflections of the hightemperature phase increased in intensity and the original reflections of the low-temperature phase disappeared altogether. In this way, the reflections of the low- and high-temperature phases coexisted as the system was driven toward the phase transition, indicating clearly that the phase transition occurred discontinuously between the lowtemperature and high-temperature forms. This behavior was detected similarly for both the ET two-component system and the ET-C4F9 copolymers. Figure 4 shows the temperature dependence of the X-ray fiber diagram taken for the uniaxially oriented ET-CF3(2.7\%) sample. The X-ray diffraction patterns, including the (00l) reflection profile, are essentially the same as those observed for the ET-C4F9 copolymers,
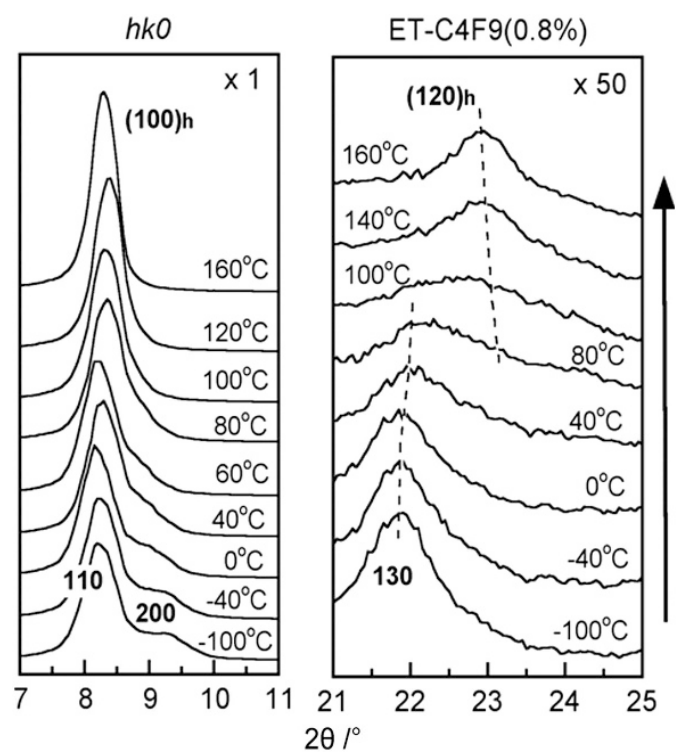

Figure 2 The temperature dependence of the $X$-ray equatorial line profile of the uniaxially oriented ET-C4F9 (0.8\%) sample, as identified from Figure 1. The Mo-K $\alpha$ line was used as the incident X-ray beam.

\section{Mo-K $\alpha$}
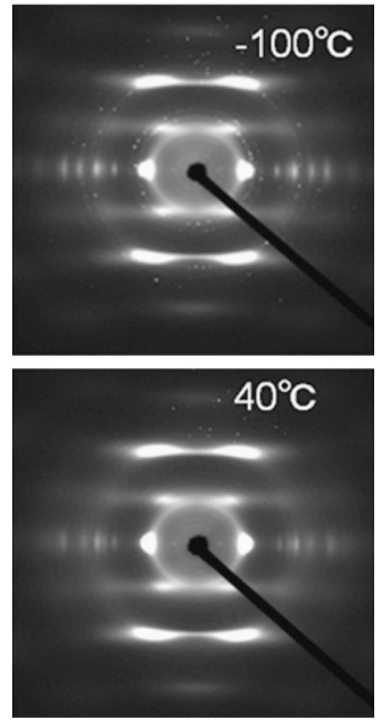
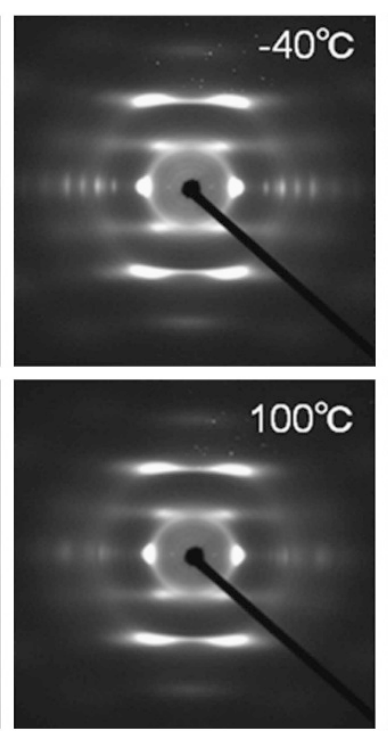

ET-C4F9(0.8\%)

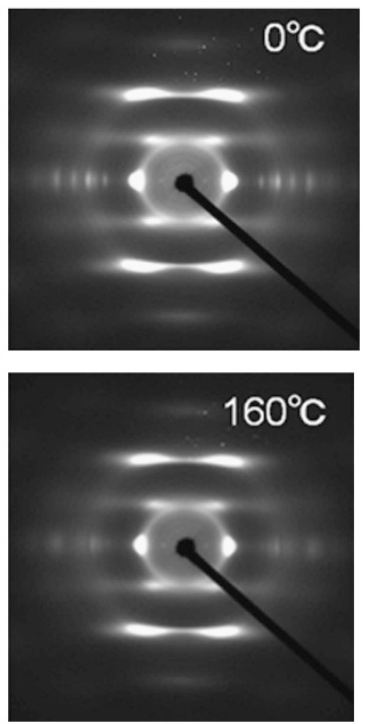

Figure 1 The X-ray fiber diagrams obtained for the uniaxially oriented ET-C4F9 (0.8\%) sample at various temperatures (the heating process). 

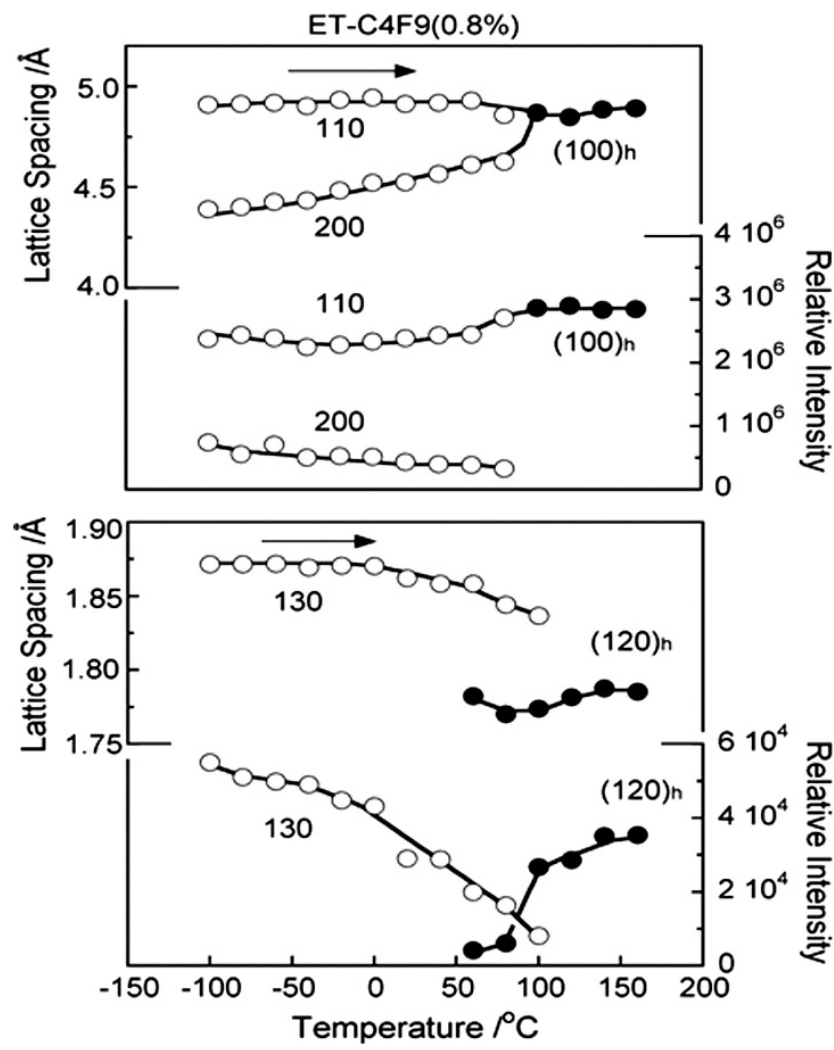

Figure 3 The temperature dependence of the lattice spacing and the diffraction intensity estimated for several $\mathrm{X}$-ray equatorial reflections of the uniaxially oriented ET-C4F9 (0.8\%) sample.

indicating essentially the same crystal structure of the unit cell as the almost perfectly disordered relative height of the neighboring chains (domains). However, the unit cell size is significantly different for the copolymers with different side groups. Figure 5 shows the equatorial line profiles obtained for the ET-CF3(2.7\%) and (4.6\%) samples, and the lattice spacings are shown in Figure 6. In the case of ETCF3(2.7\%), the inner-most reflections located at $2 \theta=8.3$ and $8.8^{\circ}$ at low temperatures correspond to the triclinic unit cell. The lattice constants projected on the equatorial plane are $a^{\prime}=9.27 \AA$ and $b^{\prime}=5.82 \AA$ and $\gamma=90^{\circ}$ at $-100^{\circ} \mathrm{C}$. The unit cell size is appreciably larger for the ET-CF3 copolymer compared with those of the ET-C4F9 copolymers and ET. This confirms the previously reported structural identification that the long $\mathrm{C}_{4} \mathrm{~F}_{9}$ side chains are expelled from the crystal lattice, whereas the short $\mathrm{CF}_{3}$ groups are included in the crystal lattice. By heating, the two reflections observed at $-100{ }^{\circ} \mathrm{C}$ merged into one at approximately $-40^{\circ} \mathrm{C}$. This tendency becomes more remarkable by further increasing the content of the third monomer unit. For example, in the case of ET-CF3(4.6\%), no reflection characteristics were detected for the low-temperature triclinic unit cell, even when the X-ray diffraction measurement was performed down to $-120^{\circ} \mathrm{C}$, where only reflections of the high-temperature, pseudo-hexagonal phase could be observed. In this way, the transition temperature of the ET-CF3 copolymers is appreciably lower than that of the ET-C4F9 samples due to the above-mentioned differences in the side groups of the crystal lattice.

\section{Polarized infrared spectra}

As already reported for the ETFE two-component copolymers, at lower temperatures, the trans-zigzag chains experienced the conformational disorder above the transition point, through the partially occurring trans-gauche (TG) exchange. ${ }^{6}$ The temperaturedependent polarized infrared spectroscopic measurements were carried out on the uniaxially oriented samples. For example,
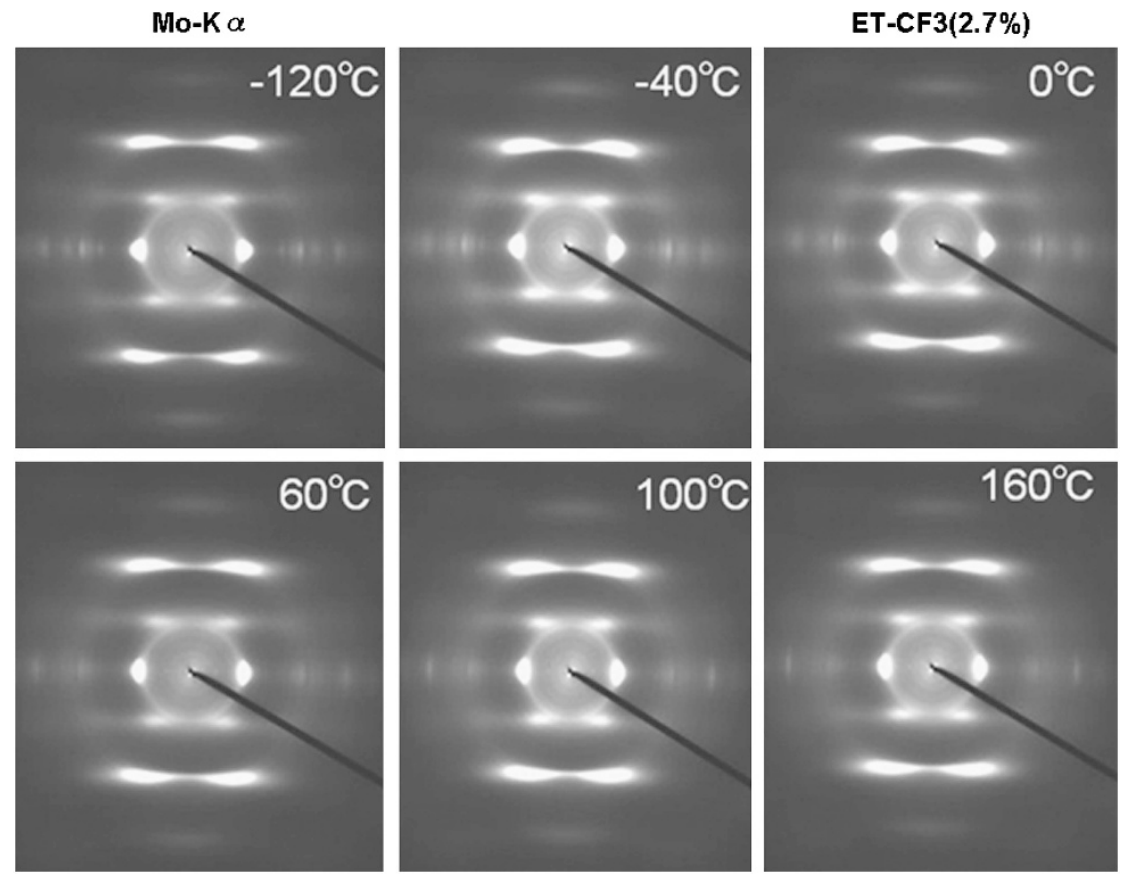

Figure 4 The X-ray fiber diagrams obtained for the uniaxially oriented ET-CF3 (2.7\%) sample at various temperatures (the heating process). 

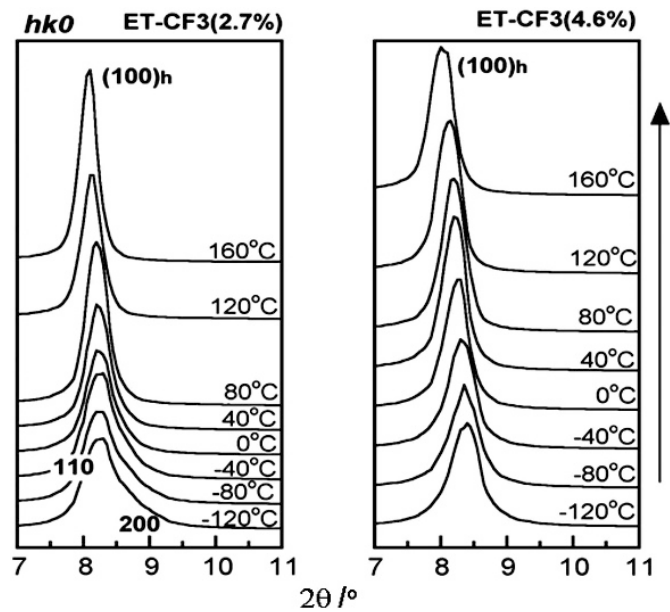

Figure 5 The temperature dependence of the $\mathrm{X}$-ray equatorial line profiles of the uniaxially oriented ET-CF3 (2.7\%) and ET-CF3 (4.6\%) samples. The Mo-K $\alpha$ line was used as the incident $\mathrm{X}$-ray beam.

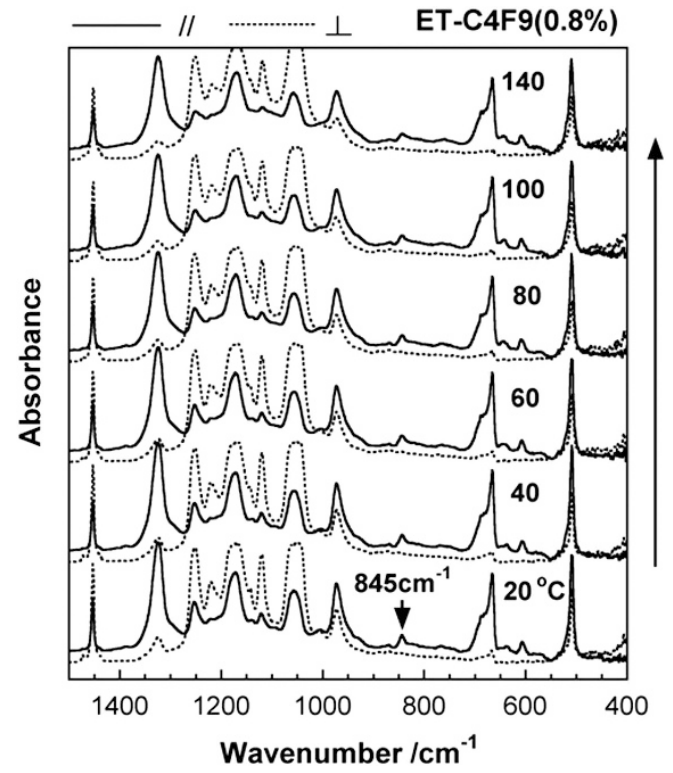

Figure 7 The temperature dependence of the polarized infrared spectra measured for the uniaxially oriented ET-C4F9 $(0.8 \%)$ sample. The solid and broken lines represent the spectra taken with the electric vector of the incident infrared beam parallel and perpendicular to the orientation direction, respectively.

Figure 7 shows the polarized infrared spectra gathered for ET$\mathrm{C} 4 \mathrm{~F} 9(0.8 \%)$ at the various temperatures in the heating process. In Figure 8, the bands at 638 and $648 \mathrm{~cm}^{-1}$, assigned to the trans-trans conformer, decreased in intensity at approximately the phase-transition point. The band at $643 \mathrm{~cm}^{-1}$ was assigned to the TG form and increased in intensity with increasing temperature. The absorbance of these bands is plotted against temperature in comparison with the wide-angle X-ray diffraction lattice spacing data (Figure 9), where the band profiles in the frequency region $630-660 \mathrm{~cm}^{-1}$ were separated into three components (two trans-trans bands and one TG band) using the software Grams/AI 8. The whole profile of the polarized infrared spectra was not significantly altered, indicating that the alltrans conformation is nearly maintained above the phase-transition temperature, but the TG conformational exchange was detected in the transition process, indicating the occurrence of some degree of chain conformational disorder. The $\mathrm{CH}_{2}$ scissoring band at $1453 \mathrm{~cm}^{-1}$ was reported to be sensitive to the thermal motion of the molecular chain. ${ }^{6}$ The half-width of this band is plotted against the temperature, as presented in Figure 9. The half-width increased with increasing temperature, indicating an enhancement of the thermally activated rotational motion of the molecular chains in the crystal lattice. The TG conformational exchange is considered to be coupled with this type of whole-chain thermal motion.

Figure 10 shows the polarized infrared spectra taken for ET$\mathrm{CF} 3(2.7 \%)$ during the heating process. Figure 11 shows the temperature dependence of infrared absorbance estimated for the trans-trans and TG bands and the half-width of the $1453-\mathrm{cm}^{-1}$ band in addition to the lattice spacings of the low-temperature 130 reflection and the high-temperature 120 reflection. In the phase-transition region, the trans-trans band decreased in intensity, whereas the TG band increased; in addition, the $1453 \mathrm{~cm}^{-1}$ became sharper. These behaviors indicate the remarkable thermal motion of molecular chains with some degree of TG conformational disordering, essentially the same as those observed for the ET and ET-C4F9 copolymers,
Figure 6 The temperature dependence of the lattice spacings estimated for several X-ray equatorial reflections of the uniaxially oriented ET-CF3 $(2.7 \%)$ and ET-CF3 (4.6\%) samples. 


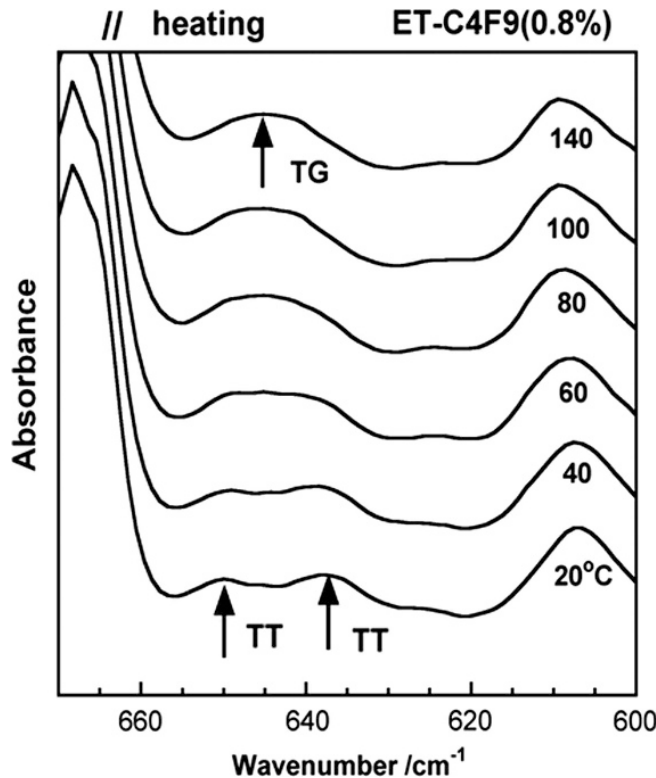

Figure 8 The temperature dependence of the polarized infrared spectra (parallel component) measured for the uniaxially oriented ET-C4F9(0.8\%) sample in the range of $600-670 \mathrm{~cm}^{-1}$.

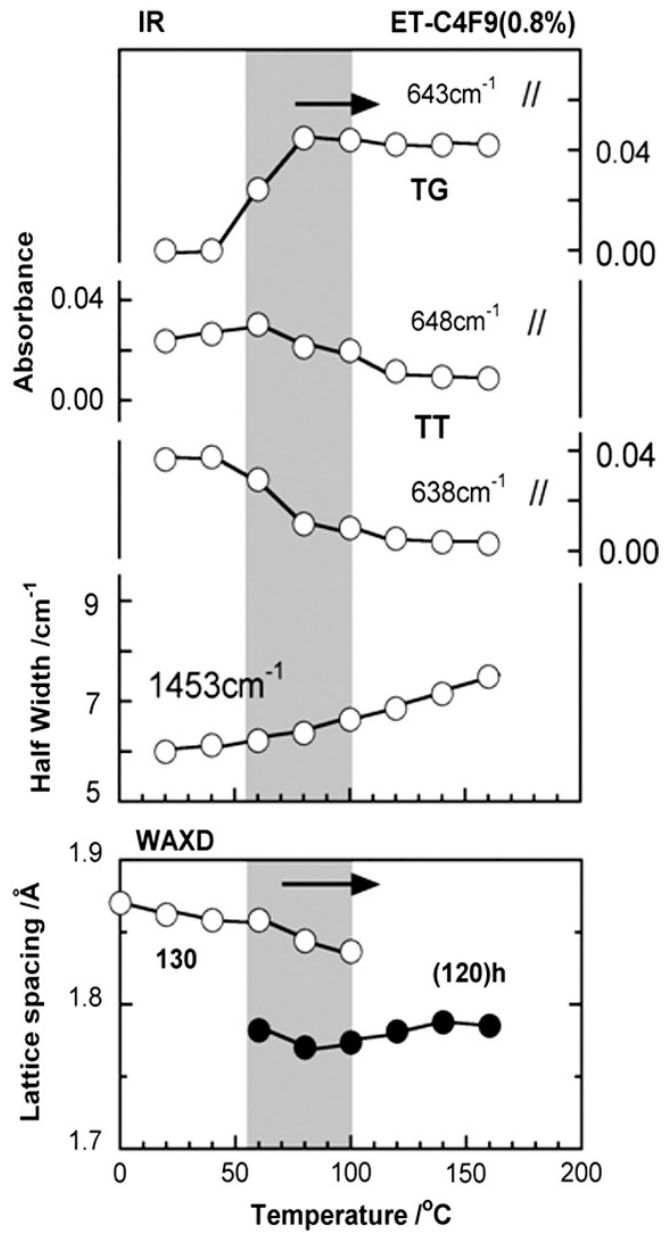

Figure 9 The temperature dependence of the infrared absorbance of the trans-gauche (TG) and trans-trans (TT) bands, the half-width of the $1453 \mathrm{~cm}^{-1}$ band and the wide-angle X-ray diffraction (WAXD) lattice spacing estimated for the uniaxially oriented ET-C4F9 (0.8\%) sample.

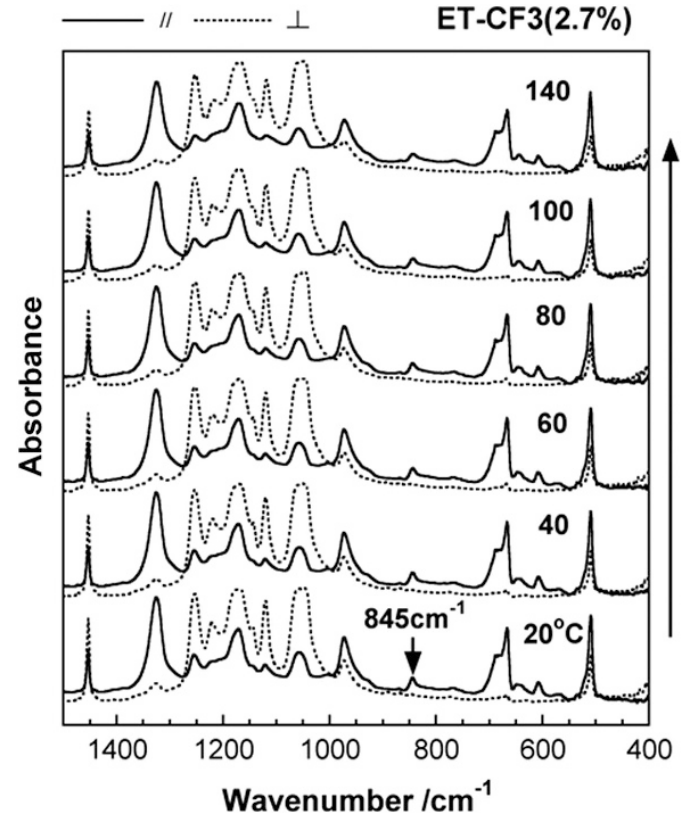

Figure 10 The temperature dependence of the polarized infrared spectra measured for the uniaxially oriented ET-CF3 (2.7\%) sample. The solid and broken lines represent the spectra taken with the electric vector of the incident infrared beam parallel and perpendicular to the orientation direction, respectively.

but in an appreciably lower phase-transition temperature region with respect to the ET-CF3 copolymer.

\section{$\mathrm{X}$-ray meridional reflections}

Figure 12 shows the temperature dependence of the half-width of the $004 \mathrm{X}$-ray reflection measured for the uniaxially oriented samples of the ET, ET-CF3(2.7\%) and ET-C4F9(1.5\%) copolymers. In the case of the ET two-component copolymer, the half-width of the reflection increased with increasing temperature and shows a deflection at approximately $90^{\circ} \mathrm{C}$. A similar phenomenon was also observed for the ET-C4F9 and ET-CF3 copolymers at the equivalent and different temperatures, respectively. These deflection points correspond well to the transition temperature regions from the low-temperature phase to the high-temperature phase. As previously reported, ${ }^{6}$ the half-width of the meridional $(\mathrm{Ool})$ reflection is sensitive to the conformational disorder as well as the thermal activity of the chains. The half value of the 004 reflection at the same temperature point (for example, at $10{ }^{\circ} \mathrm{C}$ ) is found to increase in the order of ET $\leqslant$ ET-C4F9 $<$ ET-CF3. Hence, the thermal motion of the chains in the high-temperature phase is more pronounced for the ET-CF3 copolymer compared with the ET and ET-C4F9 copolymers. As mentioned above, the unit cell size is much larger for the ET-CF3 copolymer because the short $\mathrm{CF}_{3}$ groups are incorporated into the crystal lattice, hence the chain packing is looser, which makes the thermal motion easier at lower temperatures. However, the long $\mathrm{C}_{4} \mathrm{~F}_{9}$ groups are excluded from the crystal lattice, and the chains are able to pack more compactly. As a result the unit cell size is not significantly different from that of the ET two-component sample, and the thermal motion of the molecular chains occurs in a significantly higher-temperature region.

\section{Phase diagram}

Figure 13 summarizes the transition behaviors observed for a series of ETFE copolymers by plotting the lattice spacings of the 110 and 200 


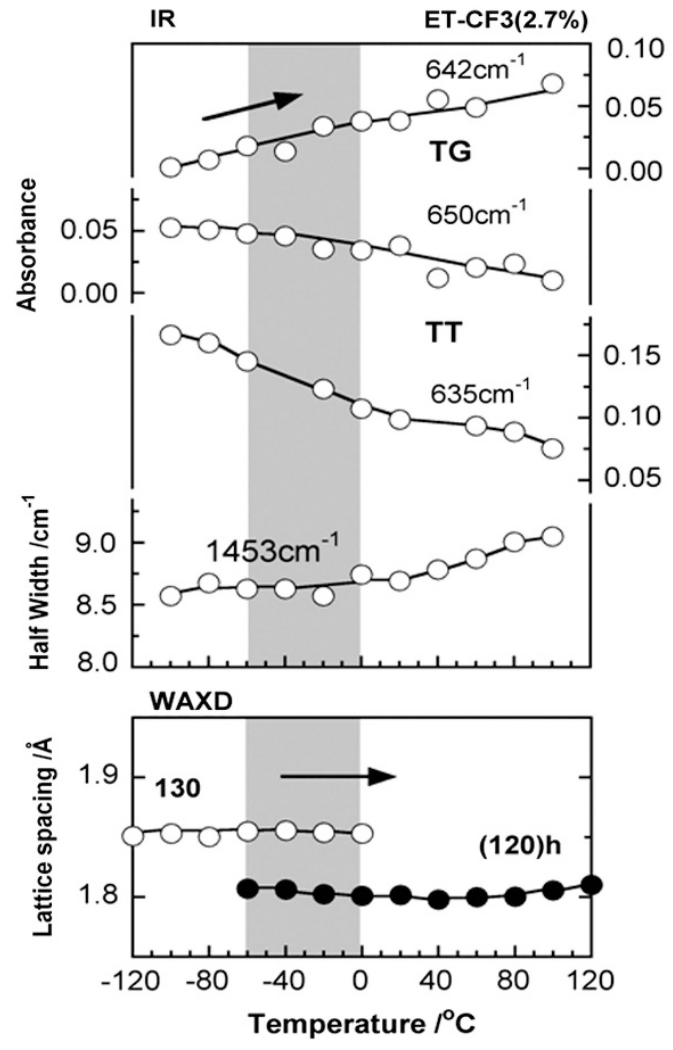

Figure 11 The temperature dependence of the infrared absorbance of the trans-gauche (TG) and trans-trans (TT) bands, the half-width of $1453 \mathrm{~cm}^{-1}$ band and the wide-angle X-ray diffraction (WAXD) lattice spacing estimated for the oriented ET-CF3 (2.7\%) sample.

reflections (Figure 13a) and 130 reflections (Figure 13b) of the lowtemperature phase, as well as the 100 and 120 reflections of the hightemperature, pseudo-hexagonal phase against the temperature. Figure 14 illustrates a distinct type of phase diagram; this phase diagram shows the phase-transition temperature plotted against the side-group content. Compared with the ET two-component copolymer, the copolymer with the short $\mathrm{CF}_{3}$ side group shows the phase transition in a much lower-temperature region. The temperature shift becomes larger with an increasing $\mathrm{CF}_{3}$ content: from 90 to $-30^{\circ} \mathrm{C}$ and below $-130{ }^{\circ} \mathrm{C}$ by increasing the content from $0 \%$ to $2.7 \%$ and $4.6 \%$, respectively. However, the copolymer with the longer $\mathrm{C}_{4} \mathrm{~F}_{9}$ side group shows the transition in the temperature region relatively close to that of the ET two-component system, even when the content is increased to $3.3 \%$. This behavior can be reasonably interpreted by investigating the relationship between the unit cell size and the transition temperature, as shown in Figure 15, where the transition point $(T c)$ is plotted against the cross-sectional area of the unit cell projected along the chain axis $\left(a^{\prime} \times b^{\prime}\right)$ estimated at $-100^{\circ} \mathrm{C}$. It is evident that the transition temperature decreases approximately linearly with the increase in cross-sectional area. This linear relationship is not applied to the ET two-component copolymer and the ETC4F9 $(0.8 \%)$ copolymer: the transition temperature is approximately the same when the third monomer unit content is $<0.8 \mathrm{~mol} \%$. When the content is increased beyond the critical point, the transition point decreases significantly. The decreasing tendency is small for the ETC4F9 copolymers, which possess a unit monomer content of 1.5 and $3.3 \mathrm{~mol} \%$; however, an increase in the effective cross-sectional area of

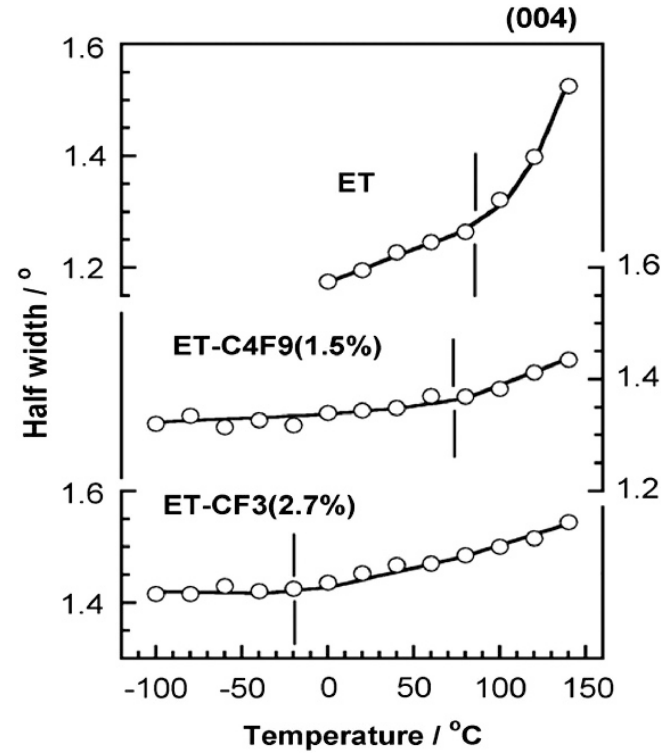

Figure 12 The temperature dependence of the half-width of X-ray (004) reflection evaluated for the ET, ET-C4F9 (1.5\%) and ET-CF3 $(2.7 \%)$ samples.
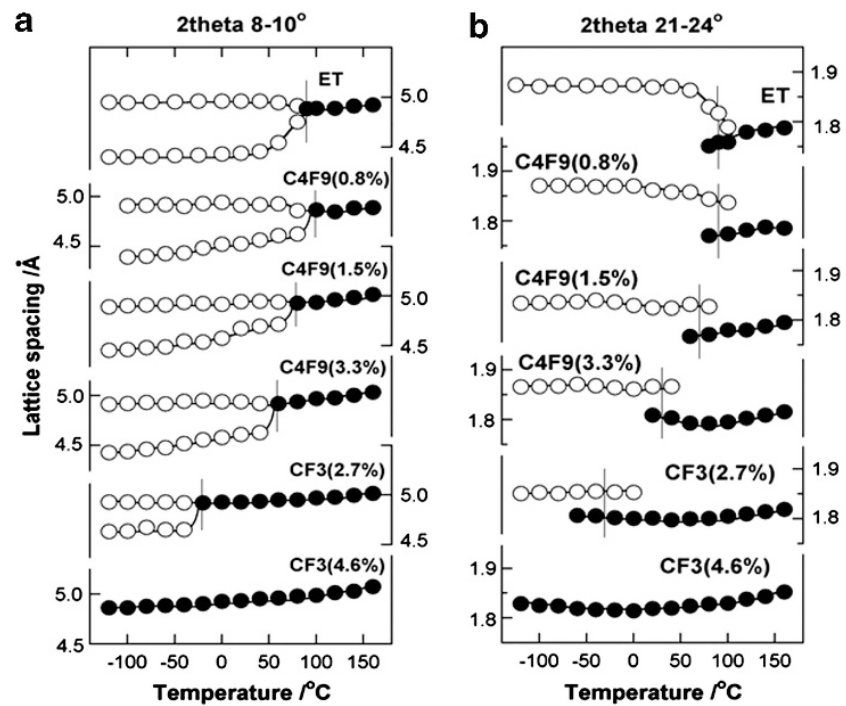

Figure 13 The temperature dependence of the lattice spacing in the scattering angle regions of (a) $2 \theta=8-10^{\circ}$ and (b) $2 \theta=21-24^{\circ}$ compared among the series of ET, ET-C4F9 and ET-CF3 copolymers.

the molecular chain can still be observed. As noted above, the long $\mathrm{C}_{4} \mathrm{~F}_{9}$ side chains are not included in the unit cell, but the unit cell area increases with the increment of the $\mathrm{C}_{4} \mathrm{~F}_{9}$ monomer content, indicating that the unit cell size is affected by the surface state of the lamella. The existence of $\mathrm{C}_{4} \mathrm{~F}_{9}$ groups on the surface may destabilize the chain packing, resulting in the increase in unit cell size. Additionally, the relatively small sift in Tc may be related to the structural characteristics of the ET-C4F9 copolymers. The situation is different with respect to the $\mathrm{CF}_{3}$ groups: the introduction of a small amount of $\mathrm{CF}_{3}$ groups enlarges the unit cell (see Figure 15). This is because the $\mathrm{CF}_{3}$ groups are included directly in the crystalline lattice. Figure 16 illustrates these structural situations schematically. 


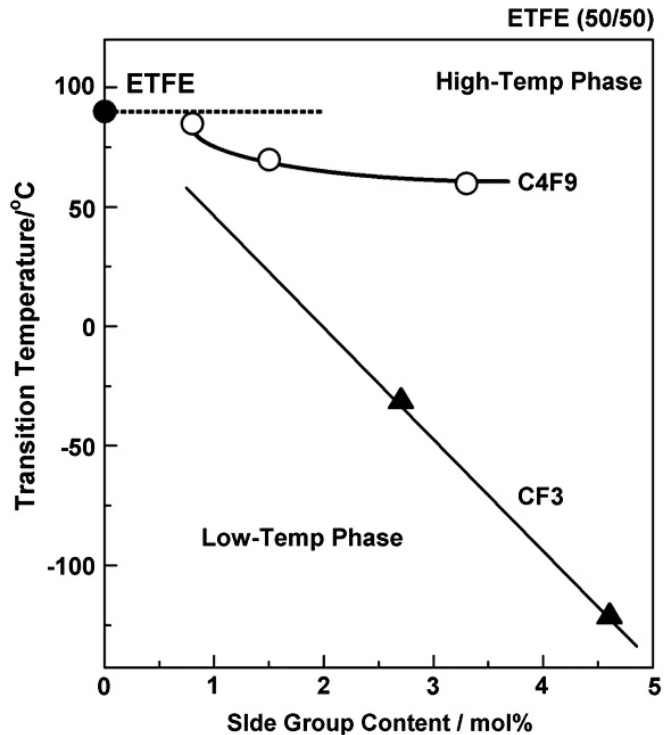

Figure 14 The transition temperatures of the ETFE copolymers plotted against the molar content of the third monomer unit. The presence of a long side group $\left(\mathrm{C}_{4} \mathrm{~F}_{9}\right)$ does not affect the transition point significantly, whereas the short $\mathrm{CF}_{3}$ group reduces the transition temperature remarkably.

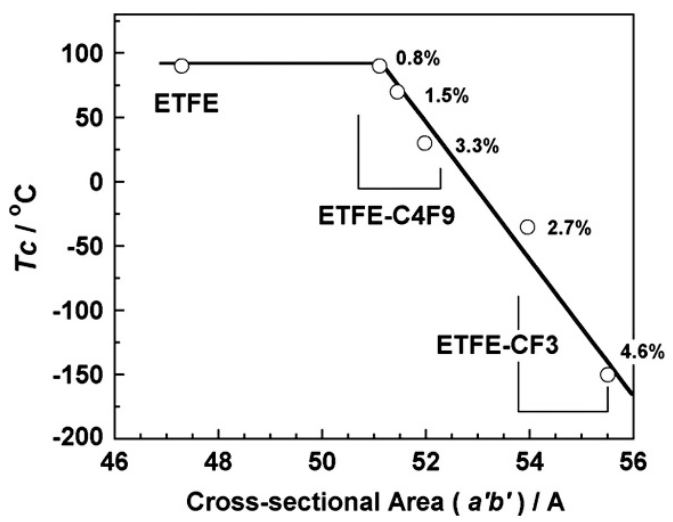

Figure 15 The correlation between the cross-sectional area of the unit cell projected along the chain axis $\left(a^{\prime} \times b^{\prime}\right)$ at $-100^{\circ} \mathrm{C}$ and the transition temperature $(T c)$ investigated for a series of ETFE copolymers.

\section{Higher-order structure}

For the various crystalline polymers, the transition to the hightemperature, pseudo-hexagonal phase induces the remarkable change in the stacked lamellar structure. For example, with the addition of a tilt angle from the draw axis, the stacked lamellae can be altered to the vertically standing lamellar structure, which possesses a much larger thickness. Typical examples of this phenomenon include the ferroelectric phase transition of vinylidene fluoride-trifluoroethylene random copolymers, ${ }^{13}$ newly discovered high-temperature phase transition of aliphatic nylons ${ }^{14,15}$ and so on. In these examples, the translational motion of chains along the chain axis is thermally activated, inducing the lamellar thickening as well as the change in tilting angle. Figure 17 shows the $2 \mathrm{D}$ small-angle X-ray scattering patterns measured for the uniaxially oriented ET, ET-C4F9(1.5\%) and ET-CF3(2.7\%) samples at room temperature. These patterns show the four-point scattering patterns and demonstrate that the lamellae are tilted approximately $20^{\circ}$ from the vertical direction. The long period

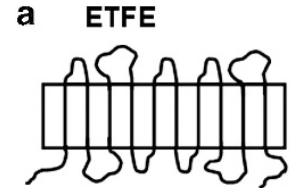

LT phase HT phase

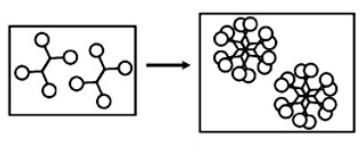

Tc $\sim 80^{\circ} \mathrm{C}$
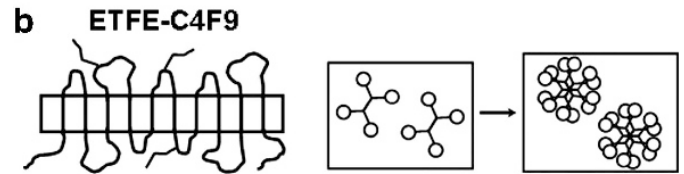

$\mathrm{Tc} \sim 60^{\circ} \mathrm{C}$

C ETFE-CF3
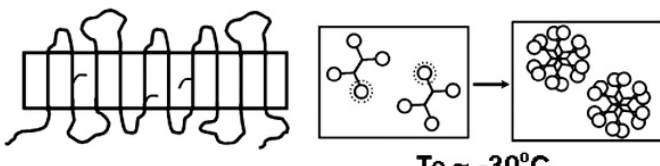

Figure 16 Schematic illustrations of the stacked lamellar structure and the phase-transition behavior: (a) the two-component ETFE, (b) the ET-C4F9 (the $\mathrm{C}_{4} \mathrm{~F}_{9}$ side chains exist on the surface of the lamella, making the chain packing unstable and resulting in a slight increase of the unit cell size as well as the decrease of $T c$ ) and (c) the ET-CF3 (the $\mathrm{CF}_{3}$ side chains are included in the crystal lattice, the unit cell is expanded and the $T c$ is reduced drastically).
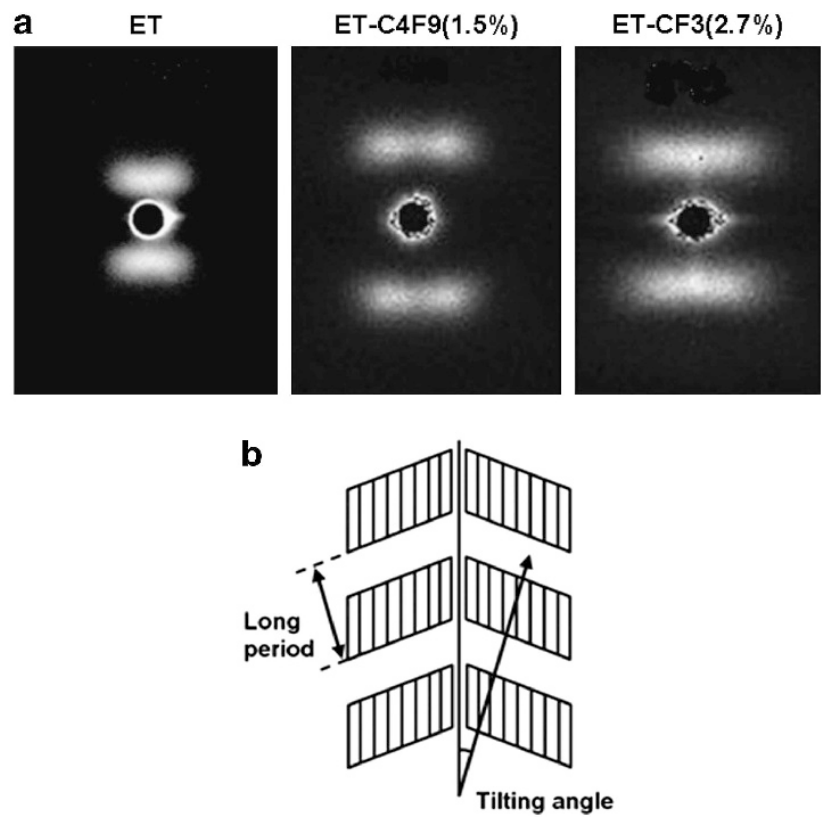

Figure 17 (a) The two-dimensional SAXS patterns taken for the uniaxially oriented ET, ET-C4F9(1.5\%) and ET-CF3(2.7\%) samples at $20^{\circ} \mathrm{C}$ and (b) a schematic illustration of the lamellar stacking structure.

is different for each of the three cases: $330 \AA$ for the ET twocomponent copolymer, $150 \AA$ for the ET-C4F9(1.5\%) copolymer and $210 \AA$ for the ET-CF3 $(2.7 \%)$ copolymer. The temperature dependence of the long period and tilt angle of the lamellae was measured for these samples and is shown in Figure 18. The vertical rod shows the phase-transition point, as revealed in Figure 13. It is clear that the transition between the low- and high-temperature phases does not 

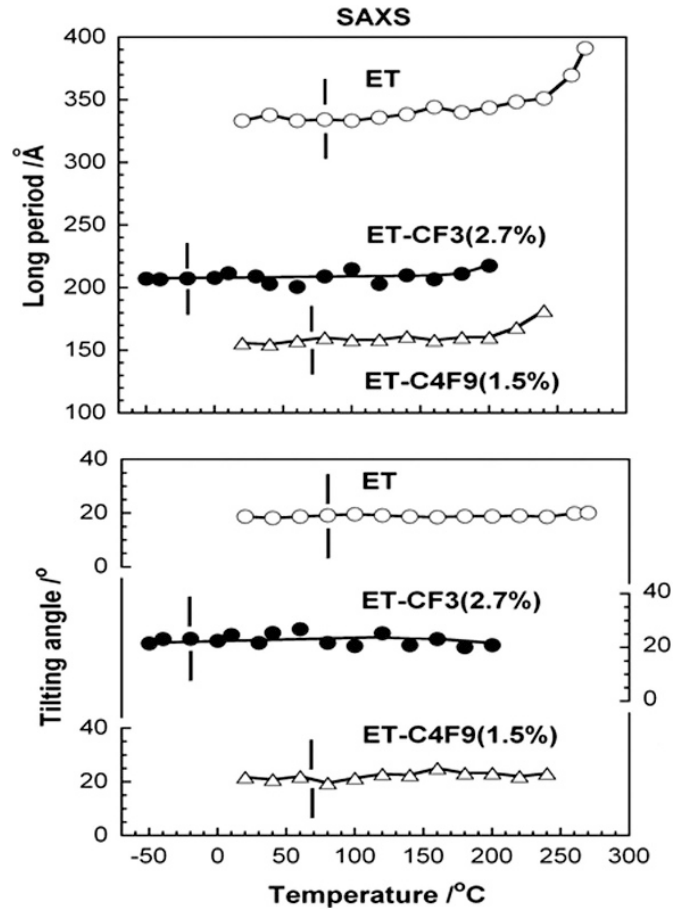

Figure 18 The temperature dependence of the long period and tilting angle estimated for the ET, ET-CF3 (2.7\%) and ET-C4F9 (1.5\%) samples.

cause any change in the higher-order structure in the ETFE copolymers, indicating that the conformational disordering and the thermal activation of the molecular chains in such low-temperature regions do not cause significant changes in the stacked lamellar structure. As frequently observed for a variety of polymers, the long period was seen to increase significantly in the higher-temperature region near the melting point, although the tilt angle did not change significantly. Currently, it is difficult to say that no change occurred in the lamellar tilt angle immediately below the melting temperature because the sample fractured as the temperature was increased further. As already demonstrated by others, ${ }^{7}$ the copolymer samples exhibit a relatively low stress cracking resistance, that is, the samples are easily broken at high temperatures when tightly constrained with a metal holder or strongly wound around a pipe. In addition, in the experiments presented in Figure 18, the samples were easily broken in the high-temperature region before the observation of remarkable change in higher-order structure could be carried out.

\section{CONCLUSIONS}

The phase-transition behavior of ETFE copolymers containing a third monomer unit component of different side-chain length has been investigated in detail by using the uniaxially oriented samples on the basis of the temperature-dependent measurements of X-ray fiber diagrams, SAXS patterns and polarized infrared spectra. The ETFEC4F9 copolymer with long side groups was found to show the orderto-disorder transition at a temperature relatively close to that observed for the pure ETFE two-component copolymer system due to the exclusion of side groups from the crystalline region. In addition, the transition point was shifted by increasing the termonomer content, although the degree of shift was not very large. However, in the copolymer samples with short $\mathrm{CF}_{3}$ side groups, the side groups were included in the crystal lattice and the unit cell expanded to accommodate a less-restricted thermal motion in the lower-temperature region. As seen from the temperature dependence of the half-widths of the X-ray 004 reflections and the infrared $1453 \mathrm{~cm}^{-1}$ band, the thermal motion of the chains was coupled with the partially occurring TG conformational exchange. The transition temperature $(T c)$ from the low-temperature to high-temperature phases was found to be intimately related to the effective crosssectional area of the molecular chains, which increased due to the existence of the side-chain groups of the third monomer unit (Figure 15). The temperature dependence of the 2D small-angle $\mathrm{X}$-ray scattering data revealed that the above-mentioned orderdisorder transition in the crystal lattice occurs without any significant change in the stacked lamellar structure, but rather, occurs in the higher-temperature region near the melting point.

In this manner, the experimental data collected for the uniaxially oriented samples provide new findings regarding the phase-transition behaviors of the ETFE copolymer and the effect of the third monomeric unit. The side groups of the third monomer units caused a possible expansion of the unit cell size, resulting in the shift of the order-disorder phase-transition temperature. However, the mechanism of unit cell expansion was different between these two cases. The existence of long side groups on the lamellar surfaces reduced the lamellar thickness and the crystal lattice became unstable, causing the expansion of the unit cell. This phenomenon is particular to the ETFE-C4F9 copolymer, and the transition point shift is rather small. However, the short $\mathrm{CF}_{3}$ groups were included in the crystal lattice and caused a more effective expansion of the unit cell size, inducing the larger shift of the transition temperature even for small contents of $\mathrm{CF}_{3}$ groups. A similar observation has been previously reported for polyethylene samples with various lengths of side groups. ${ }^{16-19}$

\section{ACKNOWLEDGEMENTS}

This study was financially supported by a MEXT 'the International Project on the Basic Research Promotion for the Development of Highly Controlled Multi-Purpose Polymer Materials' in 'the Strategic Project to Support the Formation of Research Bases at Private Universities' (2010-2014).

1 Garbuglio, C., Modena, M., Valera, M. \& Ragazzini, M. Properties of a new thermoplastic fluorinated polymer. Eur. Polym. J. 10, 91-95 (1974).

2 Nakao, T. \& Yamauchi, M. JP2007-301976A.

3 Funaki, A., Phongtamrug, S. \& Tashiro, K. Crystal structure analysis of ethylenetetrafluoroethylene alternating copolymer. Macromolecules 44, 1540-1548 (2011).

4 Iuliano, M., De Rosa, C., Guerra, G., Petraccone, V. \& Corradini, P. Structural variations in ethylene-tetrafluoroethylene copolymers as a function of composition and temperature. Macromol. Chem. 190, 827-835 (1989).

5 D'Aniello, C., De Rosa, C., Guerra, G., Petraccone, V., Corradini, P. \& Ajroldi, G. Influence of constitutional defects on polymorphic behaviour and properties of alternating ethylene-tetrafluoroethylene copolymer. Polymer (Guildf) 36, 967-973 (1995).

6 Phongtamrug, S., Tashiro, K., Arai, K., Funaki, A. \& Aida, S. Structural study of a series of ethylene-tetrafluoroethylene copolymers with various ethylene contents, part 2: phase transition behavior investigated by temperature dependenct measurements of X-Ray fiber diagrams. Polymer (Guildf) 49, 5072-5083 (2008)

7 Ukihashi, A., Yamabe, M. \& Miyake, H. JP1273050.

8 Funaki, A., Arai, K., Aida, S., Phongtamrug, S. \& Tashiro, K. Influence of third monomer on the crystal phase transition behavior of ethylene-tetrafluoroethylene copolymer. Polymer (Guildf) 49, 5497-5503 (2008).

9 Funaki, A., Takakur, T., Kato, K. \& Hamazaki, K. JP3305400.

10 Wilson, F. C. \& Starkweather, Jr. H. W. Crystal structure of an alternating copolymer of ethylene and tetrafluoroethylene. J. Polym. Sci. Polym. Phys. Ed. 11, 919-927 (1973).

11 Tanigami, T., Yamamura, K., Matsuzawa, S., Ishikawa, M., Mizoguchi, K. \& Miyasaka, K. Structural studies on ethylene-tetrafluoroethylene copolymer 1. Crystal structure. Polymer (Guildf) 27, 999-1006 (1986).

12 Phongtamrug, S., Tashiro, K., Arai, K., Funaki, A. \& Aida, S. Structural study of a series of ethylene-tetrafluoroethylene copolymers with various ethylene contents, Part 1 : structure at room temperature investigatioated for uniaxially-oriented samples by an 
organized combination of 2D-WAXD/SAXS and IR/Raman spectra. Polymer (Guildf) 49, 561-569 (2008)

13 Tashiro, K. \& Tanaka, R. Structural correlation between crystal lattice and lamellar morphology in the ferroelectric phase transition of vinylidene fluoride-trifluoroethylene copolymers as revealed by the simultaneous measurements of wide-angle and smallangle X-Ray scatterings. Polymer (Guildf) 47, 5433-5444 (2006).

14 Tashiro, K. Structural phase transition of aliphatic nylons viewed from the WAXD/SAXS and vibrational spectral measurements and molecular dynamics calculation. Chin. J. Polym. Sci. 25, 73-82 (2007)

15 Tashiro, K., Takeuchi, K., Ohta, Y., Hanesaka, M., Hashida, T., Yoshioka, Y. \& Ramesh, C. Structural phase transitions of aliphatic nylons viewed from the simultaneous measurements of WAXD and SAXS. Macromol. Symp. 242, 250-256 (2006).
16 Hay, J. N. \& Zhou, X. Q. Incorporation of branches into the crystal lattice during the solidification of linear low density polyethylene. Polymer (Guildf) 34, 1002-1005 (1993).

17 Tashiro, K. Thermodynamic and kinetic aspects of cocrystallization and phase segregation phenomena of polyethylene blends between the $D$ and $H$ Species as viewed from DSC, FTIR and synchrotron X-ray scattering. Acta. Polym. 46, 100-113 (1995).

18 Baker, A. M. E. \& Windle, A. H. An X-ray diffraction and modelling study of short branch location within the structure of polyethylene. Polymer (Guildf) 42, 681-698 (2001).

19 Watanabe, S., Sano, N., Noda, I. \& Ozaki, Y. Surface melting and lamella rearrangement process in linear low density polyethylene. J. Phys. Chem. B 113, 3385-3394 (2009) 